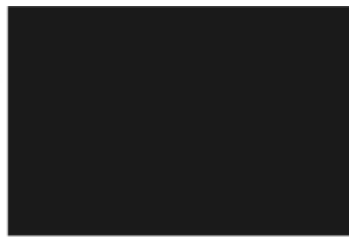

\title{
Rezensionen
}

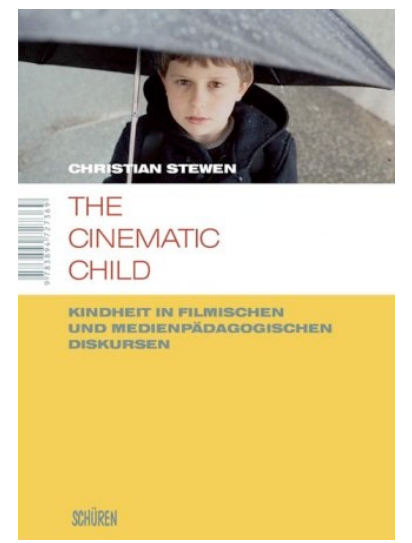

Christian Stewen

The Cinematic Child

Kindheit in filmischen und medienpädagogischen Diskursen

Marburg: Schüren, 201. 250 Seiten

ISBN: 978-3-8947-2736-9

$€ 29.90$; CHF 37.-

\section{Das Kind als cinematische Figuration}

In seiner mit dem Medius-Sonderpreis ausgezeichneten Dissertation widmet sich Christian Stewen der Frage, wie im amerikanischen Spielfilm der 1990er- und 2000er-Jahre ein spezifisches Bild von Kindheit konstruiert wird und inwieweit sich dieses Bild in medienpädagogisch motivierten Diskursen wiederfindet. Dabei verortet sich der Autor in der Tradition der foucaultschen Diskursanalyse und betrachtet Kind und Kindheit als «diskursive Produkte», wodurch das Kind den Status einer «Denkfigur» (10) annimmt, deren mediale Konstruktion es zu beleuchten gilt. Um seinem Erkenntnisinteresse nachzugehen greift Stewen "charakteristische Momente» (24) der Figuration «Kind» heraus und ordnet diese jeweils bestimmten Genres zu. Die Frage nach dem Verhältnis von Realität und Fantasie wird anhand des FantasyFilms in den Blick genommen, die Frage nach kindlicher Unschuld durch eine Analyse von Kriminalfilmen (insbesondere des Child-Witness-Dramas), spezifische Zeitvorstellungen in Bezug auf Kindheit werden an filmischen Kindheitserinnerungen und Biopics beleuchtet und anhand des Horrorfilms werden Störungen dieser Vorstellungen aufgezeigt. Im Zentrum der Untersuchung stehen also vielmehr qualitative Zuschreibungen von Erwachsenen an das Kind und weniger Kindheit als institutionalisierte Lebensphase (Honig 2008). Auch wenn die Zuordnung der kindlichen Eigenschaften zu den Genres schlüssig erscheint, bleiben die Quellen der als typisch kindlich etikettierten Eigenschaften leider offen. 
Die Untersuchung der einzelnen Eigenschaften folgt dabei schlüssig gegliedert einem einheitlichen Schema. Zunächst zeigt Stewen anhand einzelner Filme auf, wie diese den Status des Kindseins als Opposition zum Erwachsenen-Sein konstruieren; daran anschließend werden aus medienpädagogischer Perspektive nach medienreflexiven Diskursen in den Filmen gefragt und thematisch anschließende populäre Diskurse betrachtet. Das Label «medienpädagogisch» bezieht sich in diesem Kontext jedoch nicht auf die wissenschaftliche Disziplin, sondern auf ein alltagsweltliches Denkmuster bezogen auf Frage der Beziehung von Kindheit und Medialität (27). Aus der Perspektive der wissenschaftlichen Medienpädagogik lassen sich die von Stewen betrachteten Diskurszusammenhänge überwiegend als bewahrpädagogische Medienkritik verstehen.

\section{Ergebnisse}

In einem ersten Schritt widmet sich Stevens der Frage nach der Inszenierung von Fantasie im Film. Er kommt dabei zu dem Schluss, dass die dargestellten Parallelwelten oberflächlich betrachtet zwar im Gegensatz zur Realität stünden, sich bei genauerer Betrachtung jedoch als Reproduktion des real-gesellschaftlichen Kontextes offenbarten, da dessen Strukturen ein Äquivalent in der Fantasiewelt besäßen. Narrativ würde dem Kind dabei oftmals die Rolle das Erlösers zugeschrieben, der ein korrumpiertes Machsystem besiegen und die gesellschaftliche Ordnung wiederherstellen müsse. Das Kind werde hier als "aktiver (Re-)Produzent inszeniert» (47), der in einem Prozess der Initiation erwachsene Wertvorstellungen übernehme. Auf der Ebene der medienreflexiven Diskurse arbeitet Stewen heraus, dass sich Fantasie oftmals als mediale Konstruktion zu erkennen gebe, da der «Übergang von der Realität zur Fantasie [...\} als Einstieg in die mediale Präsentationsform einer Filmprojektion oder einer Fernsehdarstellung" (55) inszeniert werde, wodurch medial erzeugte Welten als Opposition zur Realität inszeniert würden. Diese Dichotomie macht Stewen auch in medienpädagogischen Diskursen aus, in welchen die Unterscheidbarkeit von Realität und Fiktion als ein wichtiger Bestandteil einer zu erlernenden Medienkompetenz gesehen wird. 
Rezensionen

In einem zweiten Schritt widmet sich Stewen den Child-Witness-Dramen und zeigt hier auf, dass die Zuschreibung des «unschuldigen Kindes» darauf beruht, dieses in einem Schutzraum unter der kontrollierenden Aufsicht eines erwachsenen Erziehers zu stellen. Erwachsene und kindliche Räume würden sich in den Filmen als klar getrennt erweisen. Im Verlauf der ChildWitness-Dramen durchbreche das Kind diese Grenze und lade somit Schuld auf sich, womit es in Kontakt mit "erwachsenen» Räumen komme. In der weiteren Inszenierung der Filme würden eindeutige Wert- und Schuldzuweisungen immer schwieriger, sodass letztlich weniger einer eindeutige unschuldig-schuldig-Dichotomie konstruiert, sondern vielmehr «der Verlust des Begriffs der Unschuld» thematisiert werde. Auf der medienreflexiven Ebene konstatiert Stewen ein Sichtbarmachen des Illusionsraum Films, indem in den Szenen der kindlichen Zeugenschaft, die Zuschauerposition des Kindes durch «eine äquivalente Positionierung des Filmzuschauers» (101) verdoppelt werde. Für die medienpädagogischen Diskurse zeigt Stewen eine Angst vor Überforderung des kindlichen Zuschauers durch ungeeignete Medientexte auf. Medien - so Stewen - komme in medienpädagogischen Diskursen «die Rolle eine Gewalttäters» (104) zu, der dank unpassender Inhalte für den Verlust der Unschuld verantwortlich sei.

Als drittes charakteristisches Moment von Kindheit macht Stewen eine spezifische zeitliche Beziehung zum Status des Erwachsenen aus, welche er anhand von Kindheitserinnerungen und Biopics genauer beschreibt. Kindheit werde in beiden Genres nur beschreibbar als «Vergangenheit von Erwachsenen» (158) und somit als "Zukunftskindheit» (159), die einzig und allein als Erklärung für das Erwachsen-Sein Bedeutung habe. In den Kindheitserinnerungen erfolge diese Rückbesinnung in der Form der Nostalgie; in den Biopics werde ein kindliches Trauma als Bedingung für das erwachsene Leben verstanden. Die Erinnerung sei dabei immer an mediale Formen meistens die Fotografie - gebunden, weshalb auf der medienreflexiven Ebene die Entwicklung von Kind zum Erwachsenen als mediale Entwicklungskette inszeniert sei. Stewen baut diesen Gedanken dahingehend aus, dass er das Kind als «filmhistorische Denkfigur» (168) und «als Metapher einer spezifischen (Film-) Geschichtsvorstellung» (169) begreift, da auch der Film im Laufe seiner Geschichte «erwachsen» geworden ist. Weitere kindliche Denkfiguren sieht Stewen in interkulturellen Diskursen, in denen einerseits unterschiedliche Gesellschaftsvorstellungen in einem Entwicklungszusammenhang gestellt würden (z.B. durch den Begriff unterentwickelter Kulturen) 
und andererseits eine spezifische Vorstellung von Kindheit als Maß für die Entwicklung einer Kultur gesehen wird. Auch die mediale Verfasstheit einer Gesellschaft werde als Zeichen von Fortschritt bzw. dessen Fehlen gedeutet. So entstehe eine medienpädagogische Paradoxie - während in der eigenen Gesellschaft die Medien oftmals für Missstände verantwortlich gemacht würden, wären sie im Kontext von Entwicklungszusammenarbeit überwiegend positiv konnotiert. An dieser Stelle muss jedoch kritisch nachgefragt werden, ob Stewen nicht die Denkfigur des Kindes überstrapaziert und nicht vielmehr eine Dominanz eines entwicklungslogischen Denkens aufzeigt, welches seit Darwin als Epistem unser Zeit gelten kann.

Im letzten Kapitel zeigt Stewen schließlich auf, wie die charakteristischen Momente von Kindheit im Horrorfilm unterlaufen werden. Die Störung bestünde darin, dass die Kinder mit erwachsen konnotierten Eigenschaften inszeniert würden, wodurch sie bedrohlich wirkten. So werde die Grenze zwischen Kindern und Erwachsenen aufgebrochen. Im Laufe der Filme würden sich die Erwachsenen des Status des Kindes immer unsicher, bis es schließlich zu einem Twist komme, indem vermeintliche Fantasien des Kindes als Wirklichkeit entlarvt und so auch erwachsene Zuschreibungen an das Kind als Projektionen ihrer eigenen Ängste erkennbar würden. Stewen theoretisiert diese Befunde, indem er das Kind als Medium denkt, das bestehende Grenzen (z.B. Leben/Tod) überwinden könne. Medienpädagogische Haltungen würden in den Filmen insofern inszeniert, als dass die kindlichen Figuren mit einem Medium verschaltet wären und die Angst vor dem Kind letztlich die Angst vor dem Medium sei.

\section{Ein Fazit}

Die Dissertation von Christian Stewen besticht durch eine theoretisch anspruchsvoll gerahmte Diskursanalyse und die Multiperspektivität der einzelnen Betrachtungen. Die Analysen der einzelnen Filme sind empirisch nachvollziehbar begründet, zeigen ein vielschichtiges Bild der Kindsdarstellungen im amerikanischen Film und rufen so den Konstruktionscharakter der «Denkfigur Kind» ins Gedächtnis.

Die Vielschichtigkeit der theoretischen Rahmungen geht leider in Einzelfällen auf Kosten der Schärfe der einzelnen Begriffe. So rahmt Stewen die Kindheitserinnerungen mit Theorien des New Historicism, welche den Konstruktionscharakter von Geschichte betonen, bezieht sich aber in den folgenden 
Ausführungen weniger auf die Konstruktion von Historizität als vielmehr auf die Lebensgeschichte der Protagonisten und den Prozess des Erinnerns, ohne die begriffliche Differenzen von Geschichte und Biographie zu reflektieren oder auf erinnerungs- und biografietheoretische Ansätze Bezug zu nehmen. Auch Kindheit und der Status des Kind-Seins werden zeitweise synonym verwendet.

Die Analyse der medienpädagogischen Diskurse reicht leider nicht an die Qualität der Filmanalysen heran. Zum einen ist das als «medienpädagogisch» bezeichnete Diskursfeld durch seine Definition als «populäres Denkmuster» sehr unscharf begrenzt, zum anderen erschweren fehlende Zitate aus dem Diskurszusammenhang die Nachvollziehbarkeit der Ergebnisse. So werden z.B. medienpädagogische Ratgeber oder Äußerungen in Talkshows benannt und inhaltlich zusammengefasst, aber nur selten mit Zitaten belegt. Häufig muss David Buckingham als Kronzeuge für den beschriebenen Diskurs herhalten, so dass der Multiperspektivität der Filmanalysen hier einer einseitigen Betrachtung weicht. Dass sich die Rekonstruktionen der medienpädagogischen Diskurse auf die bewahrpädagogische Linie beschränkt ist aus der Perspektive der wissenschaftlichen Medienpädagogik gewiss ein Makel. So entsteht der Eindruck der pädagogische Blick sei lediglich als Ergänzung der Filmanalysen und weniger als eigenständiges Feld interessant.

Dessen ungeachtet aber bleibt inm dennoch der Verdienst, im bewahrpädagogischen Diskurs dominierende Werturteile als Konstruktion aufgezeigt zu haben. Die Schlussfolgerung ein Kind sei immer auch ein Cinematic-Child ist vor diesem Hintergrund verständlich. Mit Hinblick auf die Entwicklung der neuen Medien und daraus resultierender kultureller Umwälzungen wäre es interessant, diese Denkfigur hin zu einem medialen Kind zu erweitern, in der frühere Kindsbilder als Buchkinder und die Generation der Digital Natives evtl. als Webkinder gedacht werden können. 